sonal observation. He said he thought it rational to believe that there was hypertrophy of nerves in the hypertrophied muscle of the athlete, and he did not see why the same would not apply to the pregnant uterus.

Dr. ACKER said that antipyrin, contrary to Dr. King's assertion that the hectic of phthisis could not be controlled, would bring the temperature of such cases down.

\section{CHICAGO MEDICAL SOCIETY.}

Stated Mecting, July 19,1886 .

E. J. Dofrinc, M.D., Preshont, in the Chair.

Dr. D. A. K. Steeide read a paper on

THE DIFFERENTIAI, DIAGNOSIS OF SCROTAI, TUMORS.

(See page 173.)

1)R. Christian Fenger agreed with 'Dr. Steele that gonorrhoea in itself has nothing to do with tuberculosis; that it will not cause local tuberculosis of the epididymis any more than a trauma will cause local tuberculosis anywhere else in the body. When there is tuberculosis in the body somewhere, and consequently tubercle-bacilli in the current of the blood, then it may be that the local inflammation from gonorrhceal epididynitis, just as extravasation of the blood from a trauma, favors the accumulation of tubercle-bacilli in those places. Occasionally local tuberculosis will commence in an inflamed epididymis, but in a large number of these cases of scrotal tuberculosis there has really been no gonorrhwic epididymitis preceding.

DR. W. T. Blifiel. thought that too much stress had been laid upon the history as a means of differential diagnosis between syphilitic and other enlargements of the testicle; when affirmative, the history is a valuable factor; when negative, the diagnosis must be made without regard to the history. He mentioned a case where there was not only no history of syphilis, but also a nodular enlargement of the epididymis with but slight increase in the size of the testis-features usually characteristic of tuberculosis; yet the patient's age (3I) argued so strongly against tuberculosis that it was determined to try syphilitic remedies before proposing excision; the effect was immediate and complete. Primary tuberculosis of the genital organs is evidently an infection from within-hematogenous. The idea of local infection through intercourse with a subject of uterine or ovarian tuberculosis is of course fanciful; indeed, very many of these patients are boys just beyond puberty who rarely have had intercourse. The growth of the tubercle bacilli implies some local or general predisposition of the individual; one factor in this predisposition is evidently found in the conditions developed during the rapid growth of a part. Thus in childhood the epiphyses of long bones frequently afford a nidus for the growth of the parasite, while the rudimentary sexual organs are never affected primarily; but when with puberty the genital organs begin a rapid development, they are especially prone to become the site of the infection.
'The tuberculous testicle should be excised, provided the prostate and seminal vesicles are apparently healthy, to remove the chances of further infection. While it is of course possible that there may be other and undiscovered foci of infection in various parts of the body-lymphatic glands, lungs or elsewhere-yet castration not only removes one, perhaps the only tuberculous nidus, but especially diminishes the danger of infection of the prostate and seminal vesicles-a necessarily fatal as well as agonizing affection; tuberculosis of the lungs is sometimes arrested by proper climatic and hygienic conditions; but tuberculosis of the prostate never, so far as we know, by either hygienic, medical or surgical means.

1)R. MCARTHur had little to say in regard to the differential diagnosis. In regard to scrotal tumors there was a point upon which he would like to have light, vi\%: whether Dr. Fenger would have the Society believe that tubercular testis is always the result of general tuberculosis; that is, that the tubercular bacilli are brought in the blood to the point where the local inflammation occurs. He had privately asked I)r. Belfield if such were the case, what would be the advantage of an early removal? He had removed a testicle that happened to be a tubercular onc. 'l'he man was 35 years of age, in apparently robust health, with no evidence of pillmonary or kidney affection. Dr. McArthur advised the removal of the testicle and performed the operation. On microscopic examination all the characteristics of tubercular testicle were found; within two months and a half the man died of acute tuberculosis.

Dr. Christian Fincrie said that fifteen years ago nobody would have thought of extirpating a tuberculous testicle, because we knew that after the abscess has opened and discharged for a long time, such persons, if put under favorable circumstances, given cod-liver oil, sent to the seashore or country, will recover. So far as general tuberculosis resulting from a tuberculous testicle was concerned, only about 25 per cent. of the patients, he thought, would become subject to general tuberculosis. In cases where the miliary tubercles were limited to the testicle, and all other parts of the body not as yet invaded, extirpation should be performed. It is a legitimate operation now, but has only become so within the last five years. It of course has to be limited to cases where, so far as we can find out, the tubercle is local in the epididymis. If the tuberculosis has invaded the vas deferens, it is difficult to see what good extirpation of the testicle could do except as a mere local measure. Another point in tuberculosis of the testicle makes us desire if possible to eradicate the local tuberculosis and thereby perhaps prevent the tuberculosis affecting the bladder. The patients are subject to most terrible suffering for a year or two before death when they get tuberculosis of the bladder. He did not think tuberculosis could be called local here except if it could be transmitted by cohabitation with a woman having tubercles of the uterus, much in the same way as the microbes of gonorrhcea are transferred, and this mode of infection is not proved as yet. 'The tubercles in the epididymis must origi- 
nate in the same way as local tuberculosis in the bones, by arresting and accumulation of tubercle bacilli already preexisting in the general circulation. He did not think it possible that, in the strict sense of the word, any of these tubercles could be called local.

Dr. G. C. PAori did not claim to be a specialist, but had seen many tumors of the testicles and scrotum. Tumors of the scrotum often arise in connection with diseases of the prostate glands, and with strictures. He had seen tumors of the testicle produced by affections of these organs. Another thing that gives trouble in practice is neuralgia of the testicles, which is very difficult to diagnose because the only symptom we have is the severe pain in the testicle. Another kind of tumor which has not been mentioned is schirrous, and it is often diffcult of diagnosis. Many that have been diagnosticated as schirrous have been nothıng but chronic orchitis. He once saw a testicle extirpated in the belief that it was schirrous, but it proved to be a case of chronic inflammation of the testicle.

DK. J. I. Gray read a paper on

IIL TREATMENT OF EPILEPSY HY IIGATION OF THE VERTEBRAL ARTIERIES,

first proposed by Dr. Brown, of Calcutta, in $183 \mathrm{r}$. Vertebral arteries furnish blood supply of the medulla, pons, cerebellum and posterior third of cerebrum. Real seat of local disease in epilepsy supposed to be in the medulla, especially in the vaso-motor centres and regions related thereto, as convulsive centre described by Nothnagel. Is great irritability of these centres. Attacks of epilepsy due to reflex excitement of these centres. I,igate vertebral arteries and there will be less blood sent to these diseased and hypersensitive centres. Thus is lessened ten. dency to epileptic seizures. Also, as several branches of the sympathetic nerve must be necessarily ligated at the time the arteries are, this serves to lessen vasomotor spasm in the medulla .Dr. Gray recommends tying the arteries between the atlas and axis. Operation been performed in Clicago seven times, with benefit in one case, temporary benefit in two, no benefit in two, fatal result in one, and result not known in remainder. Dr. Gray drew the following conclusions: r. ligation of vertebral arteries should take its place as a recognized procedure in cases of epilepsy in those cases where causes of attacks come from some region otuside of brain. 2. The artery should be tied as high up as possible and the ligature should include all fibres of the sympathetic nerve accompanying the vessel. 3. When side of brain first invaded by the disease can be determined, the artery of that side should be ligated, and if the lesion be bilateral both vertebral arteries should be ligated. The operation should be done not as a substitute but as an aid to other forms of treatment for the cure or relief of epilepsy.

DR. D. R. BRower in opening the discussion thanked Dr. Gray for his very able paper so full of interest and scientific importance, and said the case which he reported to the State Medical Society two years ago is now not quite so favorable. Indeed, the child has practically relapsed into the same condition she was at the time of the operation. For two or three months the change for the better in the child's condition was very manifest. In that case the ligation was made at one side, the intention was to have both arteries ligated, but such symptoms of collapse came on that it was deemed unwise to pursue the operation further, and there being so little benefit, the parents refused to have another operation. He thought with Dr. Gray that enough is before us favorable to the operation to recommend it in cases where everything else fails, and he would be disposed to press the operation in those cases where there seems to be some special disorder of the circulation in the mednlla. He thought the trouble about the treatment of epilepsy is that we have included under this name a great many diseases, he was sure there are a great many kinds of epilepsy, and he thought we do not know how to differentiate these different kinds, therefore our treatment nust be to a great extent imperfect. There seem to to be cases of epilepsy that are confined to the medulla. 'The history of some cases led him to suppose that the medulla is the seat of the disease, and in such cases it seems that the operation might prove a benefit. He did not believe that the circulation is reëstablished through the circle of Willis nearly so rapidly as Dr. Gray would have us believe. The circle of Willis is made up of minute arteries. In this particular case, there was a manifest and profound impression made upon the area of distribution of the vertebral artery for weeks, and he did not believe the collateral circulation was speedily established. He had no doubt that this operation will do good in some cases, just as any other profound im. pression will if made upon the nervous system; the cutting and destruction of the comnections through the various plexuses is beneficial, so is any extensive counter irritation. A seton added to other treatment that was only partially successful sometimes caused a wonderfully better result to follow. He had a case under observation in which the removal of a cicatrix has resulted in wonderful improvement in the patient's cundition, whether due to the extensive counter irritation produced by the operation or removal of cicatrix he did not know. He believed the operation justifiable in cases that resisted the ordinary treatment. He was an earnest advocate of the surgical treatment of epilepsy, the best results he had seen in this disease having followed the use of the surgeon's knife. Quite a number of cases had come under his observation in which the cause of the affection seemed to be in the ovarian region, and surgical interference was followed by better results than from treatment by medicine. He thought the treatment of epilepsy by pills and powders one of the most unsatisfactory things that can be undertaken, and the proportion of cases benefited by drugs exceedingly small.

Dr. Christian Fencier asked as to Alexander's operation, whether he ligated at once on both sides. The first time Dr. Fenger ligated an artery the question presented itself if it would not be dangerous to ligate them both at the same time. He ligated the arteries at Dr. Jewell's request and consequently did not take responsibility for ligating both at once. 
That question of course presents itself, and is truly interesting to see that the double simultaneous ligation of the vertebral arteries has never given rise to local brain symptoms. On the other hand, when we ligate a common carotid in case of epilepsy, we always have in mind the danger which may exist of emolition, from local cerebral enemia, and possibly death in a certain number of cases. 'The operation on the vertebral arteries is far preferable to the ligation of the carotid, if it should prove that there is no danger by the ligature of the vessels both at one time. $A \mathrm{~s}$ to the manner of operating: Alexander operated down low before the artery enters the vertebral canal, but according to Dr. Jewell's view it is necessary to ligate the vertebral arteries high up. I looked over the books of surgery and found that the method of ligating between the occiput and the atlas, and between the latter and the epistrofous, had been done on the cadaver, but not on living subjects. 'The reason why the vertebral artery had been ligated in former times was either for ancurism or wounds. There are very few instances on record, and the wounds and aneurisms were very dangerous cases, and Fischer in the German surgery of Billroth and Lurcke, published in 1880 , said that the ligature of the vertebral artery between the occiput and atlas and between the atlas and the axis is practically impossible. On the cadaver it can be done as well between the occiput and atlas as the atlas and axis, but the operation is much easier between the atlas and axis. To ligate between the occiput and the atlas I consider almost practically impossible on the living subject. The ligature between the atlas and the axis can be done; I know that Andrews ligated there, but the cases on record are few. There has not as yet been any injury to the artery, but if we should tear the artery so that we could not get hold of vessel enongh to ligate above and below, we would have much the same condition as in a traumatic aneurism of the artery. Warren, of Boston, who wrote his Memoirs of Surgery twenty years ago, gives one case of wounded artery that got well by compression, by filling up the wound, bit in most cases we know how little effective the plugging of an arterial wound is. Of thirty-four wounds of the vertebral artery only five recovered. Four of these were treated by plugging or local applications; in one only the ligature between occiput and atlas was performed by me in $188 \mathrm{I}$, and the case published in Gaillard's Jourual, July, I882. Dr. Fenger was afraid when taking up the vertebral artery of the possibility of wounding it before it is ligated.

Dr. C. W. PURDY asked Dr. Gray whether the operation was followed by diabetes, as would be expected from an operation of that kind. It was pointed out in 1849 by Claude Bernard, that traumatic injuries to this tract of the brain gives rise to diabetes in numerous instances, and since that time the course of the diabetic influence has been pretty satisfactorily mapped out. It is said to leave the brain by vaso-motor filaments with the vertebral arteries going as far as the third cervical ganglion, passing along with and surrounding the subclavian arteries, and reaching the upper and middle dorsal. A section of these nerves at any place until we reach the upper dorsal ganglion causes diabetes. Strange to say, section of the splanchnics does not cause diabetes, but he would expect to find traumatic diabetes as a result of this operation. He thought that diabetes as a result of the operation would be only temporary.

Dr. Harold N. Muyer thanked Dr. Gray for the manner in which he had presented the subject and especially for the indications which he laid down for the operation. 1)r. Moyer had uniformly bad results from the treatment of epilepsy by any operative proceedure. He had never recommended an operation for trephining that was followed by good results. $\mathrm{He}$ had once recommended the removal of the ovaries, and it was done, but was not followed by a good result. In one case coming under his observation a man was trephined three times; the first time it was followed by a cessation of the epilepsy for eighteen months; the second, for a year; the last, by no cessation. He had found medication cuite as bad; he had tried the bromides according to Hammond's method, without good results. During the last two years he had treated about one hundred cases of epilepsy and in only one case had there been any real benefit. He thought he should recommend this operation, and hoped it might not be as serious as one would be led to suppose from the deep seat of the artery, and the extensive destruction of tissue that must be made to reach it.

DR. D. R. BRower said that at the discussion which followed his paper at Springfield, a gentleman pres. ent announced that this operation was first performed in this country sixty years ago by an army surgeon, stationed in St. Jouis, upon a negro man who was his servant. The man recovered, but the case was never published. If this is so, an American surgeon was the first to essay the operation.

Dr. J. 1. GRAY in closing the discussion said that he had not had an opportunity, except in one case, to examine the urine, and in that there was a slight quantity of sugar for about a week. From all the literature he was able to examine the first operation was performed in $183 \mathrm{I}$ by Dr. Brown.

\section{CHICAGO GYNAECOLOGICAL SOCIETY.}

Forty-Seventh Regular Mecting, Friday Evening, May 28, 1886 .

'The President, Daniet, T. Nhison, M.D., in THE Chatr.

W. W. Jaggard, M.D., EmtTor.

TAIT ON ABDOMINAI, SECTION FOR PELVIC Al3SCESS.

The Secretary, Dr. Edward Warren Sawyer, read the following letter from Mr. LAwson TAIT:

My Dear Dr. Nelson:-If not too late, I should like to take part in the discussion which was entered into at the Gynacological Society of your city upon "Abdominal Section for Pelvic Abscess." My remarks, of course, are discursive and not very conclusive, because they are based upon only a very few points to which I want to draw attention. 